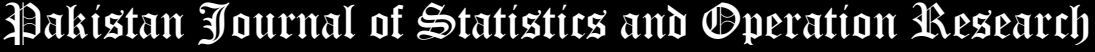

\section{Statistical Inference for Burr Type X Distribution using Geometric Process in Accelerated Life Testing Design for Time censored data}

\author{
Ahmadur Rahman ${ }^{1}$, Tabassum Naz Sindhu $^{2,3^{*}}$, Showkat Ahmad Lone ${ }^{4}$, Mustafa Kamal ${ }^{5}$ \\ * Corresponding Author \\ 1. Department of Statistics and O.R., Aligarh Muslim University, Aligarh-202002, India, \\ Kahef.ahmad@gmail.com \\ 2. Department of Statistics, Quad-i-Azam University 45320, Islamabad 44000, Pakistan, sindhuqau@gmail.com \\ 3. Department of Sciences and Humanities, FAST - National University, Islamabad, Pakistan \\ 4. Department of Statistics and O.R., Aligarh Muslim University, Aligarh-202002, India, \\ showkatmaths25@gmail.com \\ 5. College of Science and Theoretical Studies, Saudi Electronics University, Dammam, Saudi Arabia, \\ kamal19252003@gmail.com
}

\begin{abstract}
In accelerated life testing researcher generally use a life stress relationship between life characteristic and stress to estimate the parameters of failure time distributions at use condition which is just a re-parameterization of original parameters but from statistical point of view it is easy and reasonable to deal with original parameters of the distribution directly instead of developing inference for the parameters of the life stress relationship. So, an attempt is made here to estimate the parameters of Burr Type X life distribution directly in accelerated life testing by assuming that the lifetimes at increasing stress levels forms a geometric process. A mathematical model for the analysis of constant stress accelerated life testing for type-I censored data is developed and the estimates of parameters are obtained by using the maximum likelihood method. Also a Fisher information matrix is constructed in order to get the asymptotic variance and interval estimates of the parameters. Lastly, a simulation study is performed to illustrate the statistical properties of the parameters and the confidence intervals.
\end{abstract}

Key Words: Type-I censored sample; Maximum Likelihood Estimation; Reliability Function; Fisher information Matrix; Confidence Intervals; Simulation Study.

Mathematical Subject Classification: 60E05, 62E15

\section{Introduction}

In reliability analysis, researchers used to analyze time-to-failure data obtained under normal operating conditions in order to quantify the product's failure-time distribution and its associated parameters. Nowadays products has their own guarantee or warrantee schemes so the need to be tested in advance before their launch. Due to the today's highly matured technology, products are highly reliable and hence the life data is very difficult and costly to obtain at normal use conditions. This problem has motivated researchers to develop new life testing method and obtain timely information on the reliability of product components and materials. Accelerated life testing (ALT) is then adopted and widely used in manufacturing industries. Under such test settings, products are tested at higher-than-usual levels of stress to induce early failure. The objective of accelerated life inquiry is to utilize the test data to extrapolate a product's life distribution and its associated parameters at a normal stress level. The life data collected from such accelerated tests is then analyzed and extrapolated to estimate the life characteristics under normal operating conditions. Three types of stress loadings are usually applied in accelerated life tests: constant stress, step stress and linearly increasing stress. The constant stress loading, which is a time-independent test setting, has several advantages over the time- 
dependent stress loadings. For example, most products are assumed to operate at a constant stress under normal use. Therefore, a constant stress test mimics actual use. Besides, it is easier to run and to quantify a constant stress test. In the current study, we only discuss the application of constant stress in accelerated life testing.

Lam (1988) introduced the concept of geometric process in accelerated life testing to study the repair replacement problem. Lam (2007) studied the geometric process model for the multistate system and concluded a replacement policy to minimize the long run average cost per unit time.

Many authors has been studied constant stress with different types of data, For example, Yang (1994) suggested an optimal design of 4-level constant-stress ALT plans under different censoring times. Pan et al. (2011) studied a bivariate constant stress accelerated degradation test model by assuming that the copula parameter is a function of the stress level that can be described by a logistic function. Chen et al. (2012) developed the optimal design of multiple stress constant accelerated life test plan on non-rectangle test region. Watkins and John (2008) considers constant stress accelerated life tests based on Weibull distributions with constant shape and a log-linear link between scale and the stress factor which is terminated by a Type-II censoring regime at one of the stress levels. Ahmad et al. (1994) Islam and Ahmad (1994), Ahmad and Islam (1996), Ahmad et al. (2006). Ahmad (2010) discussed the optimal constant stress accelerated life test designs under periodic inspection and Type-I censoring. Huang (2011) did the analysis for exponential distribution with complete and censored data by using GP model. Zhou et al. (2012) extended the GP model for the progressive type I hybrid censored Rayleigh failure data in ALT. Kamal et al. (2013) analysed constant stress accelerated life testing for Pareto distribution with complete samples by using geometric process model. Sindhu et al. (2016) studied cumulative quantity control chart for a mixture of Rayleigh model under a Bayesian framework. Recently, Rahman, et al. (2016) studied the application of geometric process for generalized exponential distribution in accelerated life testing with complete data. Lone et al. (2016) extended this and presented a study of accelerated life testing design using geometric process for generalized exponential distribution using time constraint. Sindhu et al. (2017) explored Bayesian Analysis of two Censored Shifted Gompertz Mixture Distributions using Informative and Noninformative Priors. Sindhu et al. (2018) studied mixture of two generalized inverted exponential distributions with censored sample. Sindhu et al. (2019) investigated parameter and reliability estimation of inverted Maxwell mixture model.

This article focused on the maximum likelihood method for estimating the parameters of Burr Type X distribution. This work was conducted for constant stress ALT for Burr Type X using geometric process with type-I censored data. The confidence intervals for parameters are also obtained by using the asymptotic properties of normal distribution. In the last, the statistical properties of estimates and confidence intervals are examined through a simulation study.

\section{Model Description and Test Procedure 2.1 Geometric Process}

A GP describes a stochastic process $\left\{X_{n}, n=1,2, \ldots\right\}$ such that $\left\{\omega^{n-1} X_{n}, n=1,2, \ldots\right\}$ formulates a renewal process where $\omega>0$, is real valued and is called the ratio of the GP. It is obvious that a GP is stochastically increasing if $0<$ $\omega<1$ and stochastically decreasing if $\omega>1$. Hence, the GP is a natural way to scrutinize data from a series of events with trend. For more details about GP and its properties see Braun et al. [10]. The probability density function of $X_{1}$ in GP such that $\left\{X_{n}, n=1,2, \ldots\right\}$ is $f(x)$ with mean $\mu$ and variance $\sigma^{2}$, subsequently the probability density function of $X_{n}$ will be $\omega^{n-1} f\left(\omega^{n-1} x\right)$ with mean $\mu / \omega^{n-1}$ and variance $\sigma^{2} / \omega^{2(n-1)}$.

\subsection{Burr Type X Distribution}

Burr (1942) introduced twelve different forms of cumulative distribution functions for modeling data. Among those twelve distribution functions, Burr-Type X and Burr-Type XII received the maximum attention. There is a thorough analysis of Burr-Type XII distribution in Rodriguez (1977), see also Wingo (1993) for a nice account of it. In this paper, we consider the two-parameter Burr-Type X distribution. Two-parameter Burr-Type X distribution has the following cumulative distribution function (CDF);

$F(x, \alpha, \lambda)=\left(1-\exp \left\{-\left(\frac{x}{\lambda}\right)^{2}\right\}\right)^{\alpha}, x, \alpha$ and $\lambda>0$.

The probability density function of Burr -Type $\mathrm{X}$ Distribution is given by

$f(x, \alpha, \lambda)=\frac{2 \alpha x}{\lambda^{2}} \exp \left\{-\left(\frac{x}{\lambda}\right)^{2}\right\}\left(1-\exp \left\{-\left(\frac{x}{\lambda}\right)^{2}\right\}\right)^{\alpha-1}, x, \alpha$ and $\lambda>0$.

The survival function of Burr- Type X Distribution has the following form

$S(x, \alpha, \lambda)=1-\left(1-\exp \left\{-\left(\frac{x}{\lambda}\right)^{2}\right\}\right)^{\alpha}, x, \alpha$ and $\lambda>0$.

The failure rate or hazard rate is given by 
$h(x, \alpha, \lambda)=\frac{\frac{2 \alpha x}{\lambda^{2}} \exp \left\{-\left(\frac{x}{\lambda}\right)^{2}\right\}\left(1-\exp \left\{-\left(\frac{x}{\lambda}\right)^{2}\right\}\right)^{\alpha-1}}{1-\left(1-\exp \left\{-\left(\frac{x}{\lambda}\right)^{2}\right\}\right)^{\alpha}}, x, \alpha$ and $\lambda>0$.

Where $\alpha$ and $\lambda$ are shape and scale parameters, respectively. Burr-Type X distribution were studied by Sartawi and Abu-Salih (1991), Jaheen (1996), Ahmad et al. (1997), Raqab (1998). Surles and Padgett (2001) studies and observed that the Burr-Type X distribution can be used quite effectively in modeling strength data and also modeling general lifetime data.

\subsection{Assumptions}

(i) Suppose that an accelerated life test with $s$ increasing stress levels in which a random sample of $n$ identical items is placed under each stress level and start to operate at the same time. Let $x_{k i}, i=1,2, \ldots, n, k=1,2, \ldots, s$ denote observed failure time of $i t h$ test item under $k$ th stress level. Whenever an item fails, it will be removed from the test and the test is terminated at a pre-specified censoring time $t$ at each stress level and the exact failure times $x_{k i} \leq t$ of items are observed.

(ii) The product life under each stress level follows Burr-Type $\mathrm{X}$ distribution denoted byBurr-type $\mathrm{X}(x, \alpha, \lambda)$.

(iii) The scale parameter is a $\log$-linear function of stress. That is, $\log \lambda_{k}=a+b S_{k}$ where $a$ and $b$ are unknown parameters depending on the nature of the product and the test method.

(iv) Let random variables $X_{0}, X_{1}, X_{2}, \ldots, X_{S}$ denote the lifetimes under each stress level, where $X_{0}$ denotes item's lifetime under the design stress at which items will operate ordinarily and sequence $\left(X_{k}, k=1,2, \ldots, s\right)$ forms a geometric process with ratio $\omega>0$.

The assumption (iv) can be shown by the following theorem assuming that there is a log linear relationship between a life and stress (assumption (iii)).

Theorem: In ALT, if the stress level is increasing with a constant difference then the life times of items forms a GP under each stress level. That is, If $S_{k+1}-S_{k}$ is constant for $k=1,2, \ldots, s-1$ then $\left(X_{k}, k=1,2, \ldots, s\right)$ forms a GP.

Proof: From assumption (iv), we get

$\log \left(\frac{\lambda_{k+1}}{\lambda_{k}}\right)=b\left(S_{k+1}-S_{k}\right)=b \Delta S$

This shows that the increased stress levels form an arithmetic sequence with a constant difference $\Delta S$. Now the above equation can be written as

$\left(\frac{\lambda_{k+1}}{\lambda_{k}}\right)=e^{b \Delta S}=\omega$ (say)

It is clear from (6(a)) that

The lifetime pdf of an item at the $k t h$ stress level is

$$
\lambda_{k}=\frac{1}{\omega} \lambda_{k-1}=\frac{1}{\omega^{2}} \lambda_{k-2}=\ldots=\frac{1}{\omega^{k}} \lambda
$$

$$
\begin{array}{r}
f_{X_{k}}(x)=\frac{2 \alpha x}{\lambda_{k}{ }^{2}} \exp \left\{-\left(\frac{x}{\lambda_{k}}\right)^{2}\right\}\left(1-\exp \left\{-\left(\frac{x}{\lambda_{k}}\right)^{2}\right\}\right)^{\alpha-1}, \\
f_{X_{k}}(x)=\left(\frac{\omega^{k}}{\lambda}\right)^{2} 2 \alpha x \exp \left\{-\left(\frac{\omega^{k} x}{\lambda}\right)^{2}\right\}\left(1-\exp \left\{-\left(\frac{\omega^{k} x}{\lambda}\right)^{2}\right\}\right)^{\alpha-1} .
\end{array}
$$

And the cdf is given as

$F_{x_{k}}(x)=\left(1-\exp \left\{-\left(\frac{\omega^{k} x}{\lambda}\right)^{2}\right\}\right)^{\alpha}$

This implies that

$$
f_{X_{k}}(x)=\omega^{k} f_{X_{0}}\left(\omega^{k} x\right)
$$

From the definition of GP and from expression (6(b)) it is clear that, if density functions of $X_{0}$ is $f_{X_{0}}(x)$, then the pdf of $X_{k}$ will be given by $\omega^{k} f_{X_{0}}\left(\omega^{k} x\right), k=1,2, \ldots, s$. Therefore, it is clear that lifetimes under a sequence of arithmetically increasing stress levels form a GP with ratio $\omega$.

\section{Maximum Likelihood Estimation}

The maximum likelihood method of estimation is used because ML method is very robust and gives the estimates of parameter with good statistical properties. In this method, the estimates of parameters are those values which maximize 
the sampling distribution of data. However, ML estimation method is very simple for one parameter distributions but its implementation in ALT is mathematically more intense and, generally, estimates of parameters do not exist in closed form, therefore, numerical techniques such as Newton Method, Some computer programs are used to compute them. Let the test at each stress level is terminated at time $t$ and only $x_{k i} \leq t$ failure times are observed. Assume that $r_{k}(\leq n)$ failures at the $k^{\text {th }}$ stress level are observed before the test is suspended and $\left(n-r_{k}\right)$ units are still survived the entire test without failing. Now the likelihood function for constant stress ALT with Type I censored data using GP at one of the stress level is given by

$$
\begin{aligned}
& L(\lambda, \omega, \alpha)= \frac{n !}{(n-r) !}\left\{( \frac { \omega ^ { k } } { \lambda } ) ^ { 2 r _ { k } } ( 2 \alpha ) ^ { r _ { k } } \prod _ { i = 1 } ^ { r _ { k } } x _ { k ( i ) } \operatorname { e x p } \{ - ( \frac { \omega ^ { k } x _ { k ( i ) } } { \lambda } ) ^ { 2 } \} \left(1-\exp \left\{-\left(\frac{\left.\left.\left.\left.\omega^{k} x_{k(i)}\right)^{2}\right\}\right)^{\alpha-1}\right\}}{\lambda}\right\}\right.\right.\right. \\
& \times\left\{1-\left(1-\exp \left\{-\left(\frac{\omega^{k} t}{\lambda}\right)^{2}\right\}\right)^{\alpha}\right\} . \\
& L(\lambda, \omega, \alpha)=\prod_{k=1}^{s-r_{k}}[\left.\frac{n !}{(n-r) !}\left\{\left(\frac{\omega^{k}}{\lambda}\right)^{2 r_{k}}(2 \alpha)^{r_{k}} \prod_{i=1}^{r_{k}} x_{k(i)} \exp \left\{-\left(\frac{\omega^{k} x_{k(i)}}{\lambda}\right)^{2}\right\}\left(1-\exp \left\{-\left(\frac{\omega^{k} x_{k(i)}}{\lambda}\right)^{2}\right\}\right)^{\alpha-1}\right\}\right] . \text { The log- } \\
&\left.\times\left\{1-\left(1-\exp \left\{-\left(\frac{\omega^{k} t}{\lambda}\right)^{2}\right\}\right)^{\alpha}\right\}^{n-r_{k}}\right\}
\end{aligned}
$$

likelihood function corresponding above expression takes the form

$$
=\sum_{k=1}^{s}\left[\begin{array}{c}
l=\ln L_{k}(\lambda, \omega, \alpha) \\
+(\alpha-1) \sum_{i=1}^{r_{k}} \ln \left(1-\exp \left\{-\left(\frac{\omega^{k} x_{k(i)}}{\lambda}\right)^{2}\right\}\right)+\left(n-r_{k}\right) \ln \left\{1-\left(1-\exp \left\{-\left(\frac{\omega^{k} t}{\lambda}\right)^{2}\right\}\right)^{\alpha}\right\} \\
+\left(\alpha r_{k} \ln \omega-2 r_{k} \ln \lambda+r_{k} \ln \alpha+r_{k} \ln \alpha+r_{k} \ln 2+\sum_{i=1}^{r_{k}} \ln x_{k(i)}-\left(\frac{\omega^{k}}{\lambda}\right)^{2} \sum_{i=1}^{r_{k}} x_{k(i)}\right.
\end{array} .\right.
$$

MLE's of $\lambda, \omega$ and $\alpha$ are obtained by solving the following normal equations

$$
\begin{aligned}
& \frac{\partial l}{\partial \lambda}=\sum_{i=1}^{S}\left\{\frac{2 E^{\alpha-1} D\left(n-r_{k}\right) t^{2} \omega^{2 k} \alpha}{B \lambda^{3}}-\frac{2 r_{k}}{\lambda}+\left(\frac{2 \omega^{2 k}}{\lambda^{3}}\right) \sum_{i=1}^{r_{k}}\left(x_{k(i)}-(\alpha-1) \frac{C}{A} x^{2}{ }_{k(i)}\right)\right\}=0 . \\
& \frac{\partial l}{\partial \omega}=\sum_{i=1}^{S}\left\{\frac{2 k r_{k}}{\omega}-\frac{2 k \omega^{2 k-1}}{\lambda^{2}}\left(\frac{E^{\alpha-1} D\left(n-r_{k}\right) t^{2} \alpha}{B}-\sum_{i=1}^{r_{k}} x_{k(i)}+(\alpha-1) \frac{C}{A} \sum_{i=1}^{r_{k}} x^{2}{ }_{k(i)}\right)\right\}=0 . \\
& \frac{\partial l}{\partial \alpha}=\sum_{i=1}^{S}\left\{\frac{r_{k}}{\alpha}-\frac{\left(n-r_{k}\right) E^{\alpha} \ln E}{B}+\sum_{i=1}^{r_{k}} \ln A\right\}=0 .
\end{aligned}
$$

Where

$$
\begin{aligned}
& A=\left\{1-\exp \left(-\left(\frac{\omega^{k} x_{k(i)}}{\lambda}\right)^{2}\right)\right\}, B=1-\left\{1-\exp \left(-\left(\frac{\omega^{k} t}{\lambda}\right)^{2}\right)\right\}^{\alpha}, C=\exp \left(-\left(\frac{\omega^{k} x_{k(i)}}{\lambda}\right)^{2}\right), \\
& D=\exp \left(-\left(\frac{\omega^{k} t}{\lambda}\right)^{2}\right), E=1-D, B=1-E^{\alpha} .
\end{aligned}
$$

Equations (8), (9) and (10) are nonlinear; therefore, it is very difficult to obtain a solution in closed form. So, NewtonRaphson method is used to solve these equations simultaneously to obtain $\hat{\alpha}, \widehat{\omega}$ and $\hat{\lambda}$.

\section{Asymptotic Confidence Interval Estimates}

The large sample theory states that the ML estimators are consistent and normally distributed under some appropriate regularity conditions. It is impossible to obtain the exact confidence intervals (CIs) because the above estimates of parameters are not in closed form. So instead of exact CIs, asymptotic CIs based on the asymptotic normal distribution of ML estimators are obtained. The Fisher-information matrix composed of the negative second partial derivatives of $\log$ likelihood function. The elements of the Fisher Information matrix are given below 


$$
\begin{aligned}
& \frac{\partial^{2} l}{\partial \omega^{2}} \\
& =\sum_{i=1}^{s}\left\{\begin{array}{c}
-\frac{2 k r_{k}}{\omega^{2}}+\left(n-r_{k}\right)\left(\frac{4 E^{\alpha-1} D k^{2} t^{4} \omega^{4 k-2} \alpha}{B \lambda^{4}}\right)\left(1-E^{-1} D(\alpha-1)-\frac{E^{\alpha-1} D \alpha}{B}-\frac{(2 k-1)}{2 k t^{2} \omega^{2 k}}\right) \\
-\frac{2 k(2 k-1) \omega^{2 k-2} \sum_{i=1}^{r_{k}} x_{k(i)}}{\lambda^{2}}+(\alpha-1) \sum_{i=1}^{r_{k}}\left(\frac{2 C k \omega^{2 k-2} x^{2}{ }_{k(i)}}{A \lambda^{2}}\right)\left\{(2 k-1)-\frac{2 k \omega^{2 k} x^{2}{ }_{k(i)}}{\lambda^{2}}-\frac{2 C k \omega^{2 k} x^{2}{ }_{k(i)}}{A \lambda^{2}}\right\}
\end{array}\right\} \\
& \frac{\partial^{2} l}{\partial \alpha^{2}}=\sum_{i=1}^{s}\left\{-\frac{r_{k}}{\alpha^{2}}-\frac{\left(n-r_{k}\right) E^{\alpha} \ln E}{B}\left(\frac{2 E^{\alpha}}{B}+1\right)\right\} \\
& \frac{\partial^{2} l}{\partial \lambda^{2}}=\sum_{i=1}^{s}\left\{\begin{array}{c}
\frac{2 E^{\alpha-1} D\left(n-r_{k}\right) t^{4} \omega^{4 k} \alpha}{B \lambda^{6}}\left(2-2 E^{-1} D(\alpha-1)-\frac{2 E^{\alpha-1} D \alpha}{B}-\frac{3 \lambda^{2}}{t^{2} \omega^{2 k}}\right)+\frac{2 r_{k}}{\lambda^{2}}-6\left(\frac{\omega^{2 k}}{\lambda^{4}}\right) \sum_{i=1}^{r_{k}} x_{k(i)} \\
+(\alpha-1) \sum_{i=1}^{r_{k}}\left(\frac{2 C \omega^{2 k} x^{2}{ }_{k(i)}}{A \lambda^{4}}\right)\left\{3-\frac{2 \omega^{2 k} x^{2}{ }_{k(i)}}{\lambda^{2}}-\frac{2 C \omega^{2 k} x^{2}{ }_{k(i)}}{A \lambda^{2}}\right\}
\end{array}\right\} \\
& \frac{\partial^{2} l}{\partial \alpha \partial \omega}=\sum_{i=1}^{s}\left\{\frac{2 E^{\alpha-1}\left(n-r_{k}\right) D k t^{2} \omega^{2 k-1}}{B \lambda^{2}}\left(-1-\alpha \ln E-\frac{E^{\alpha} \alpha \ln E}{B}\right)+\sum_{i=1}^{r_{k}} x_{k(i)}^{2}\left(\frac{2 C k \omega^{2 k-1}}{A \lambda^{2}}\right)\right\} \\
& \frac{\partial^{2} l}{\partial \lambda \partial \omega}=\sum_{i=1}^{s}\left\{\begin{array}{l}
\frac{4 E^{\alpha-1}\left(n-r_{k}\right) D k t^{4} \omega^{4 k-1} \alpha}{B \lambda^{5}}\left(D(\alpha-1) E^{-1}-1+\frac{E^{\alpha-1} D \alpha}{B}\right)+\left(n-r_{k}\right)\left(\frac{4 E^{\alpha-1} D k t^{2} \omega^{2 k-1} \alpha}{B \lambda^{3}}\right) \\
+\sum_{i=1}^{r_{k}} x_{k(i)}\left(\frac{4 k \omega^{2 k-1}}{\lambda^{3}}\right)+(\alpha-1) \sum_{i=1}^{r_{k}}\left(\frac{4 C k \omega^{2 k-1} x^{2}{ }_{k(i)}}{A \lambda^{3}}\right)\left\{-1+\frac{\omega^{2 k} x^{2}{ }_{k(i)}}{\lambda^{2}}+\frac{C \omega^{2 k} x^{2}{ }_{k(i)}}{A \lambda^{2}}\right\}
\end{array}\right\} \\
& \frac{\partial^{2} l}{\partial \lambda \partial \alpha}=\sum_{i=1}^{s}\left\{\frac{2 E^{\alpha-1}\left(n-r_{k}\right) D t^{2} \omega^{2 k}}{B \lambda^{3}}\left(1+\alpha \ln E+\frac{E^{\alpha} \alpha \ln E}{B}\right)-\sum_{i=1}^{r_{k}}\left(\frac{2 C \omega^{2 k} x^{2}{ }_{k(i)}}{A \lambda^{3}}\right)\right\}
\end{aligned}
$$

The Fisher Information matrix is

$$
I(\Theta)=-E\left[\begin{array}{ccc}
\frac{\partial^{2} l}{\partial \lambda^{2}} & \frac{\partial^{2} l}{\partial \lambda \partial \omega} & \frac{\partial^{2} l}{\partial \lambda \partial \alpha} \\
\frac{\partial^{2} l}{\partial \omega \partial \lambda} & \frac{\partial^{2} l}{\partial \omega^{2}} & \frac{\partial^{2} l}{\partial \omega \partial \alpha} \\
\frac{\partial^{2} l}{\partial \alpha \partial \lambda} & \frac{\partial^{2} l}{\partial \alpha \partial \omega} & \frac{\partial^{2} l}{\partial \alpha^{2}}
\end{array}\right]
$$

where $\Theta=(\lambda, \omega, \alpha)$.

The variance covariance matrix can be written as

$$
\Sigma=\left\{-E\left[\begin{array}{ccc}
\frac{\partial^{2} l}{\partial \lambda^{2}} & \frac{\partial^{2} l}{\partial \lambda \partial \omega} & \frac{\partial^{2} l}{\partial \lambda \partial \alpha} \\
\frac{\partial^{2} l}{\partial \omega \partial \lambda} & \frac{\partial^{2} l}{\partial \omega^{2}} & \frac{\partial^{2} l}{\partial \omega \partial \alpha} \\
\frac{\partial^{2} l}{\partial \alpha \partial \lambda} & \frac{\partial^{2} l}{\partial \alpha \partial \omega} & \frac{\partial^{2} l}{\partial \alpha^{2}}
\end{array}\right]\right\}^{-1}=\left[\begin{array}{ccc}
A \operatorname{var}(\hat{\lambda}) & A \operatorname{cov}(\hat{\lambda} \widehat{\omega}) & A \operatorname{cov}(\hat{\lambda} \hat{\alpha}) \\
A \operatorname{cov}(\widehat{\omega} \hat{\lambda}) & A \operatorname{var}(\widehat{\omega}) & A \operatorname{cov}(\widehat{\omega} \hat{\alpha}) \\
A \operatorname{cov}(\hat{\alpha} \hat{\lambda}) & A \operatorname{cov}(\hat{\alpha} \widehat{\omega}) & A \operatorname{var}(\hat{\alpha})
\end{array}\right] .
$$

The $100(1-\gamma) \%$ asymptotic confidence interval for $\lambda, \omega$, and $\alpha$ are then given respectively as

$$
\hat{\lambda} \pm Z_{1-\frac{\gamma}{2}} \sqrt{\operatorname{var}(\hat{\lambda})}, \widehat{\omega} \pm Z_{1-\frac{\gamma}{2}} \sqrt{\operatorname{var}(\widehat{\omega})}, \hat{\alpha} \pm Z_{1-\frac{\gamma}{2}} \sqrt{\operatorname{var}(\hat{\alpha})}
$$

\section{Simulation Study}

Simulation study is a numerical technique which conducts experiments on the computer. We simulate pseudo random data to study the properties of the parameters. It models the condition which is assumed in the study and elaborate the behavior of the model and function. The simulation process of the current study is as follows:

1. Generate a pseudo sample u from uniform distribution over interval $[0,1]$. 
2. Now we use inverse cdf method to transform eq $(6(\mathrm{c}))$ in terms of $\mathrm{u}$ as follows

$$
x_{k i}=\lambda * \sqrt{(}\left(-\log \left(1-u^{\frac{1}{\alpha}}\right) / \omega^{k}\right.
$$

3. Different samples of sizes 25, 50, 75, 100, 125 and 150 have been obtained from the Burr Type X distribution.

4. The values of the parameters and numbers of the stress levels are chosen to be $\alpha=2, \lambda=2.9, \omega=1.5$ and $\mathrm{s}=4,6$ and 8.

5. By using the optim() function of R software, we obtain ML estimates, mean squared error (MSE), relative error $(\mathrm{RE})$, relative absolute bias (RAB) and lower and upper bound of $95 \%$ and $99 \%$ confidence intervals for the said sample sizes.

Table 1: Simulation results of Burr type X distribution using GP for $\alpha=2, \lambda=2.9, \omega=1.5$ and $\mathrm{s}=4$

\begin{tabular}{|c|c|c|c|c|c|c|c|c|}
\hline Sample & Estimate & Mean & SE & $\sqrt{\mathrm{MSE}}$ & RAB & $\mathrm{RE}$ & $\begin{array}{l}\text { Lower } \\
\text { Bound }\end{array}$ & $\begin{array}{l}\text { Upper } \\
\text { Bound }\end{array}$ \\
\hline \multirow{3}{*}{25} & $\alpha$ & 2.1093 & 0.1158 & 0.1129 & 0.0546 & 0.0564 & $\begin{array}{l}1.8821 \\
1.8103\end{array}$ & $\begin{array}{l}2.3364 \\
2.4083\end{array}$ \\
\hline & $\lambda$ & 2.6289 & 0.4157 & 0.4052 & 0.0934 & 0.1397 & $\begin{array}{l}1.8141 \\
1.5563\end{array}$ & $\begin{array}{l}3.4437 \\
3.7015\end{array}$ \\
\hline & $\omega$ & 2.0760 & 0.5943 & 0.5792 & 0.3840 & 0.3861 & $\begin{array}{l}0.9111 \\
0.5426\end{array}$ & $\begin{array}{l}3.2409 \\
3.6094\end{array}$ \\
\hline \multirow{3}{*}{50} & $\alpha$ & 2.1409 & 0.1694 & 0.1651 & 0.0704 & 0.0825 & $\begin{array}{l}1.8087 \\
1.7037\end{array}$ & $\begin{array}{l}2.4731 \\
2.5781\end{array}$ \\
\hline & $\lambda$ & 2.6946 & 0.2509 & 0.2446 & 0.0708 & 0.0843 & $\begin{array}{l}2.2027 \\
2.0471\end{array}$ & $\begin{array}{l}3.1865 \\
3.3421\end{array}$ \\
\hline & $\omega$ & 2.1024 & 0.6187 & 0.6031 & 0.4016 & 0.4020 & $\begin{array}{l}0.8895 \\
0.5059\end{array}$ & $\begin{array}{l}3.3152 \\
3.6989\end{array}$ \\
\hline \multirow{3}{*}{75} & $\alpha$ & 2.1502 & 0.1758 & 0.1716 & 0.0751 & 0.0858 & $\begin{array}{l}1.8054 \\
1.6964\end{array}$ & $\begin{array}{l}2.4950 \\
2.6040\end{array}$ \\
\hline & $\lambda$ & 2.6715 & 0.3055 & 0.2981 & 0.0787 & 0.1028 & $\begin{array}{l}2.0727 \\
1.8832 \\
\end{array}$ & $\begin{array}{l}3.2704 \\
3.4599\end{array}$ \\
\hline & $\omega$ & 2.0840 & 0.6001 & 0.5856 & 0.3893 & 0.3904 & $\begin{array}{l}0.9077 \\
0.5356\end{array}$ & $\begin{array}{l}3.2603 \\
3.6324\end{array}$ \\
\hline \multirow{3}{*}{100} & $\alpha$ & 2.1638 & 0.1752 & 0.1707 & 0.0819 & 0.0853 & $\begin{array}{l}1.8204 \\
1.7118\end{array}$ & $\begin{array}{l}2.5072 \\
2.6158\end{array}$ \\
\hline & $\lambda$ & 2.6620 & 0.3694 & 0.3601 & 0.0820 & 0.1241 & $\begin{array}{l}1.9378 \\
1.7087 \\
\end{array}$ & $\begin{array}{l}3.3862 \\
3.6152 \\
\end{array}$ \\
\hline & $\omega$ & 2.0972 & 0.6158 & 0.6002 & 0.3981 & 0.4001 & $\begin{array}{l}0.8902 \\
0.5084 \\
\end{array}$ & $\begin{array}{l}3.3042 \\
3.6860 \\
\end{array}$ \\
\hline \multirow{3}{*}{125} & $\alpha$ & 2.1247 & 0.1322 & 0.1288 & 0.0623 & 0.0644 & $\begin{array}{l}1.8655 \\
1.7835\end{array}$ & $\begin{array}{l}2.3838 \\
2.4658\end{array}$ \\
\hline & $\lambda$ & 2.7362 & 0.2157 & 0.2103 & 0.0564 & 0.0725 & $\begin{array}{l}2.3133 \\
2.1795 \\
\end{array}$ & $\begin{array}{l}3.1591 \\
3.2929 \\
\end{array}$ \\
\hline & $\omega$ & 2.0965 & 0.6125 & 0.5970 & 0.3977 & 0.3980 & $\begin{array}{l}0.8959 \\
0.5161 \\
\end{array}$ & $\begin{array}{l}3.2972 \\
3.6769 \\
\end{array}$ \\
\hline \multirow{3}{*}{150} & $\alpha$ & 2.1387 & 0.1467 & 0.1429 & 0.0693 & 0.0714 & $\begin{array}{l}1.8511 \\
1.7602 \\
\end{array}$ & $\begin{array}{l}2.4263 \\
2.5172 \\
\end{array}$ \\
\hline & $\lambda$ & 2.6267 & 0.3877 & 0.3778 & 0.0942 & 0.1303 & $\begin{array}{l}1.8668 \\
1.6264 \\
\end{array}$ & $\begin{array}{l}3.3867 \\
3.6270 \\
\end{array}$ \\
\hline & $\omega$ & 2.0757 & 0.5938 & 0.5788 & 0.3838 & 0.3858 & $\begin{array}{l}0.9118 \\
0.5436 \\
\end{array}$ & $\begin{array}{l}3.2396 \\
3.6078 \\
\end{array}$ \\
\hline
\end{tabular}


Table 2: Simulation results of Burr type X distribution using GP for $\alpha=2, \lambda=2.9, \omega=1.5$ and $\mathrm{s}=6$

\begin{tabular}{|c|c|c|c|c|c|c|c|c|}
\hline Sample & Estimate & Mean & SE & $\sqrt{\mathrm{MSE}}$ & RAB & RE & $\begin{array}{l}\text { Lower } \\
\text { Bound }\end{array}$ & $\begin{array}{l}\text { Upper } \\
\text { Bound }\end{array}$ \\
\hline \multirow{3}{*}{25} & $\alpha$ & 2.0359 & 0.0385 & 0.0375 & 0.0179 & 0.0187 & $\begin{array}{l}1.9604 \\
1.9365\end{array}$ & $\begin{array}{l}2.1115 \\
2.1354\end{array}$ \\
\hline & $\lambda$ & 2.8400 & 0.0838 & 0.0817 & 0.0206 & 0.0281 & $\begin{array}{l}2.6757 \\
2.6237\end{array}$ & $\begin{array}{l}3.0044 \\
3.0564\end{array}$ \\
\hline & $\omega$ & 1.9534 & 0.4841 & 0.4718 & 0.3022 & 0.3145 & $\begin{array}{l}1.0045 \\
0.7044\end{array}$ & $\begin{array}{l}2.9023 \\
3.2024\end{array}$ \\
\hline \multirow{3}{*}{50} & $\alpha$ & 2.0300 & 0.0313 & 0.0305 & 0.0150 & 0.0152 & $\begin{array}{l}1.9685 \\
1.9490\end{array}$ & $\begin{array}{l}2.0915 \\
2.1110\end{array}$ \\
\hline & $\lambda$ & 2.8594 & 0.0424 & 0.0413 & 0.0139 & 0.0142 & $\begin{array}{l}2.7762 \\
2.7498\end{array}$ & $\begin{array}{l}2.9427 \\
2.9690\end{array}$ \\
\hline & $\omega$ & 1.8786 & 0.3945 & 0.3845 & 0.2524 & 0.2563 & $\begin{array}{l}1.1053 \\
0.8607\end{array}$ & $\begin{array}{l}2.6520 \\
2.8966\end{array}$ \\
\hline \multirow{3}{*}{75} & $\alpha$ & 2.0309 & 0.0320 & 0.0312 & 0.0154 & 0.0156 & $\begin{array}{l}1.9680 \\
1.9482\end{array}$ & $\begin{array}{l}2.0937 \\
2.1136\end{array}$ \\
\hline & $\lambda$ & 2.8602 & 0.0409 & 0.0398 & 0.0136 & 0.0137 & $\begin{array}{l}2.7800 \\
2.7547 \\
\end{array}$ & $\begin{array}{l}2.9405 \\
2.9658 \\
\end{array}$ \\
\hline & $\omega$ & 1.8937 & 0.4093 & 0.3990 & 0.2625 & 0.2660 & $\begin{array}{l}1.0913 \\
0.8375\end{array}$ & $\begin{array}{l}2.6961 \\
2.9499 \\
\end{array}$ \\
\hline \multirow{3}{*}{100} & $\alpha$ & 2.0306 & 0.0314 & 0.0306 & 0.0153 & 0.0153 & $\begin{array}{l}1.9690 \\
1.9495\end{array}$ & $\begin{array}{l}2.0921 \\
2.1116 \\
\end{array}$ \\
\hline & $\lambda$ & 2.8588 & 0.0422 & 0.0411 & 0.0141 & 0.0142 & $\begin{array}{l}2.7760 \\
2.7498\end{array}$ & $\begin{array}{l}2.9416 \\
2.9678\end{array}$ \\
\hline & $\omega$ & 1.8871 & 0.3976 & 0.3876 & 0.2580 & 0.2584 & $\begin{array}{l}1.1076 \\
0.8611\end{array}$ & $\begin{array}{l}2.6665 \\
2.9131\end{array}$ \\
\hline \multirow{3}{*}{125} & $\alpha$ & 2.0312 & 0.0321 & 0.0313 & 0.0156 & 0.0156 & $\begin{array}{l}1.9682 \\
1.9483\end{array}$ & $\begin{array}{l}2.0942 \\
2.1141\end{array}$ \\
\hline & $\lambda$ & 2.8579 & 0.0432 & 0.0421 & 0.0145 & 0.0145 & $\begin{array}{l}2.7731 \\
2.7463\end{array}$ & $\begin{array}{l}2.9426 \\
2.9694\end{array}$ \\
\hline & $\omega$ & 1.8959 & 0.4069 & 0.3966 & 0.2639 & 0.2644 & $\begin{array}{l}1.0983 \\
0.8460 \\
\end{array}$ & $\begin{array}{l}2.6936 \\
2.9459 \\
\end{array}$ \\
\hline \multirow{3}{*}{150} & $\alpha$ & 2.0319 & 0.0327 & 0.0319 & 0.0159 & 0.0159 & $\begin{array}{l}1.9676 \\
1.9473 \\
\end{array}$ & $\begin{array}{l}2.0961 \\
2.1164 \\
\end{array}$ \\
\hline & $\lambda$ & 2.8569 & 0.0441 & 0.0430 & 0.0148 & 0.0148 & $\begin{array}{l}2.7704 \\
2.7430\end{array}$ & $\begin{array}{l}2.9435 \\
2.9709\end{array}$ \\
\hline & $\omega$ & 1.9046 & 0.4157 & 0.4051 & 0.2697 & 0.2701 & $\begin{array}{l}1.0898 \\
0.8321\end{array}$ & $\begin{array}{l}2.7194 \\
2.9772\end{array}$ \\
\hline
\end{tabular}


Table 3: Simulation results of Burr type X distribution using GP for $\alpha=2, \lambda=2.9, \omega=1.5$ and $\mathrm{s}=8$

\begin{tabular}{|c|c|c|c|c|c|c|c|c|}
\hline Sample & Estimate & Mean & SE & $\sqrt{\mathrm{MSE}}$ & RAB & $\mathrm{RE}$ & $\begin{array}{l}\text { Lower } \\
\text { Bound }\end{array}$ & $\begin{array}{l}\text { Upper } \\
\text { Bound }\end{array}$ \\
\hline \multirow{3}{*}{25} & $\alpha$ & 2.0128 & 0.0325 & 0.0307 & 0.0125 & 0.0143 & $\begin{array}{l}1.9678 \\
1.9473\end{array}$ & $\begin{array}{l}2.0987 \\
2.1014\end{array}$ \\
\hline & $\lambda$ & 2.8703 & 0.0653 & 0.0632 & 0.0195 & 0.0209 & $\begin{array}{l}2.6964 \\
2.6187\end{array}$ & $\begin{array}{l}2.9918 \\
3.0285 \\
\end{array}$ \\
\hline & $\omega$ & 1.8437 & 0.3761 & 0.3748 & 0.2352 & 0.2410 & $\begin{array}{l}1.0131 \\
0.7254\end{array}$ & $\begin{array}{l}2.8376 \\
3.0958\end{array}$ \\
\hline \multirow{3}{*}{50} & $\alpha$ & 2.0275 & 0.0285 & 0.0269 & 0.0118 & 0.0127 & $\begin{array}{l}1.9845 \\
1.9689\end{array}$ & $\begin{array}{l}2.0903 \\
2.1102\end{array}$ \\
\hline & $\bar{\lambda}$ & 2.8628 & 0.0552 & 0.0534 & 0.0137 & 0.0142 & $\begin{array}{l}2.8013 \\
2.7814\end{array}$ & $\begin{array}{l}2.9547 \\
2.9958\end{array}$ \\
\hline & $\omega$ & 1.8267 & 0.3651 & 0.3628 & 0.2252 & 0.2285 & $\begin{array}{l}1.1443 \\
0.9725\end{array}$ & $\begin{array}{l}2.6548 \\
2.6087\end{array}$ \\
\hline \multirow{3}{*}{75} & $\alpha$ & 2.0173 & 0.0273 & 0.0254 & 0.0104 & 0.0131 & $\begin{array}{l}1.9832 \\
1.9632\end{array}$ & $\begin{array}{l}2.0738 \\
2.0986\end{array}$ \\
\hline & $\lambda$ & 2.8813 & 0.0409 & 0.0398 & 0.0128 & 0.0136 & $\begin{array}{l}2.8123 \\
2.7769 \\
\end{array}$ & $\begin{array}{l}2.9384 \\
2.9518 \\
\end{array}$ \\
\hline & $\omega$ & 1.7331 & 0.3261 & 0.3252 & 0.2345 & 0.2387 & $\begin{array}{l}1.0982 \\
0.9567\end{array}$ & $\begin{array}{l}2.4684 \\
2.4859\end{array}$ \\
\hline \multirow{3}{*}{100} & $\alpha$ & 2.0183 & 0.0295 & 0.0284 & 0.0123 & 0.0138 & $\begin{array}{l}1.9813 \\
1.9536\end{array}$ & $\begin{array}{l}2.0765 \\
2.0894 \\
\end{array}$ \\
\hline & $\lambda$ & 2.8672 & 0.0440 & 0.0427 & 0.0135 & 0.0149 & $\begin{array}{l}2.7927 \\
2.7815\end{array}$ & $\begin{array}{l}2.9329 \\
2.9516\end{array}$ \\
\hline & $\omega$ & 1.7471 & 0.3432 & 0.3418 & 0.2256 & 0.2287 & $\begin{array}{l}1.1384 \\
0.9818\end{array}$ & $\begin{array}{l}2.5186 \\
2.5637\end{array}$ \\
\hline \multirow{3}{*}{125} & $\alpha$ & 2.0139 & 0.0317 & 0.0313 & 0.0116 & 0.0128 & $\begin{array}{l}1.9751 \\
1.9493\end{array}$ & $\begin{array}{l}2.0789 \\
2.0915\end{array}$ \\
\hline & $\lambda$ & 2.8954 & 0.0382 & 0.0365 & 0.0133 & 0.0148 & $\begin{array}{l}2.7891 \\
2.7628 \\
\end{array}$ & $\begin{array}{l}2.9299 \\
2.9573 \\
\end{array}$ \\
\hline & $\omega$ & 1.7350 & 0.3289 & 0.3268 & 0.2371 & 0.2403 & $\begin{array}{l}1.1289 \\
0.9736\end{array}$ & $\begin{array}{l}2.4762 \\
2.5934\end{array}$ \\
\hline \multirow{3}{*}{150} & $\alpha$ & 2.0102 & 0.0269 & 0.0248 & 0.0120 & 0.0132 & $\begin{array}{l}1.9847 \\
1.9658\end{array}$ & $\begin{array}{l}2.0764 \\
2.0885\end{array}$ \\
\hline & $\bar{\lambda}$ & 2.8813 & 0.0375 & 0.0361 & 0.0126 & 0.0142 & $\begin{array}{l}2.8016 \\
2.7786\end{array}$ & $\begin{array}{l}2.9320 \\
2.9587\end{array}$ \\
\hline & $\omega$ & 1.6990 & 0.3486 & 0.3470 & 0.2341 & 0.2452 & $\begin{array}{l}1.0785 \\
0.9698\end{array}$ & $\begin{array}{l}2.3982 \\
2.5298\end{array}$ \\
\hline
\end{tabular}

Conducting the above algorithm, the average values of SE, MSE, RAB and RE are obtained using 1000 replications to avoid randomness. The results presented in Table 1-3 are based on different sample sized with parameter values $\alpha=2, \lambda=2.9, \omega=1.5$ and $s=4,6$ and 8 to investigate the performance of the MLEs of the model parameters.

It is observed from Table 1-3 that in almost all cases the estimates of parameters in Table 3 give smaller MSEs, RABs and REs compared to those in Table 1 and 2. In all cases the MSEs of the MLEs of the parameters in all three Table (1-3) decreases as the sample size increases.

\section{Conclusion and Future Work:}

In this article, we have considered the likelihood estimation of Burr-Type X distribution parameters using geometric process in accelerated life testing for the two different stress levels. The MLEs of the parameters are obtained numerically using the Newton-Raphson method and their performances are evaluated and discussed in terms of SE, MSE, RAB and RE. From the simulation results it is easy to find that maximum likelihood estimates have good statistical properties. As a future work, Bayesian inference using geometric process in accelerated life testing assuming in this article will be considered. 


\section{References}

1. Ahmad, N., Islam, A., Kumar, R. and Tuteja, R. K. (1994). Optimal Design of Accelerated Life Test Plans Under Periodic Inspection and Type I Censoring: The Case of Rayleigh Failure Law. South African Statistical Journal, 28, 27-35.

2. Ahmad, N. and Islam, A. (1996). Optimal accelerated life test designs for Burr type XII distributions under periodic inspection and type I censoring. Naval Research Logistics, 43, 1049-1077.

3. Ahmad, K. E., Fakhry, M. E. and Jaheen, Z. F. (1997). Empirical Bayes estimation of $P(Y<X)$ and characterization of Burr-type X model. Journal of Statistical Planning and Inference, 64, 297-308.

4. Ahmad, N., Islam, A. and Salam, A. (2006). Analysis of optimal accelerated life test plans for periodic inspection: The case of Exponentiated Weibull failure model. International Journal of Quality \& Reliability Management, 23(8), 1019-1046.

5. Ahmad, N. (2010). Designing Accelerated Life Tests for Generalized Exponential Distribution with Loglinear Model. International Journal of Reliability and Safety, 4 (2/3), 238-264.

6. Burr, I. W. (1942). Cumulative frequency distribution. Annals of Mathematical Statistics, 13, 215-232.

7. Braun, W. J., Li, W. and Zhao, Y. Q. (2005). Properties of the geometric and related processes. Naval Research Logistics, 52 (7), 607-616.

8. Chen, W., Gao, L., Liu, J., Qian, P. and Pan, J. (2012). Optimal design of multiple stress constant accelerated life test plan on non-rectangle test region. Chinese Journal of Mechanical Engineering, 25 (6), 1231-1237. http://dx.doi.org/10.3901/CJME.2012.06.1231

9. Huang, S. (2011). Statistical inference in accelerated life testing with geometric process model, Master's thesis, San Diego State University.

10. Islam, A. and Ahmad, N. (1994). Optimal design of accelerated life test plan for Weibull distribution under periodic inspection and Type I censoring. Microelectronics Reliability, 34 (9), 1459- 1468.

11. Jaheen, Z. F. (1996). Empirical Bayes estimation of the reliability and failure rate functions of the Burr type $\mathrm{X}$ failure model. Journal of Applied Statistical Sciences, 3, 281-288.

12. Kamal, M., Zarrin, S. and Islam, A. (2013). Accelerated life testing design using geometric process for Pareto distribution. International Journal of Advanced Statistics and Probability, 1 (2), 25-31.

13. Lam, Y. (1988). Geometric Process and Replacement Problem. Acta Mathematicae Applicatae Sinica, 4 (4), 366-377.

14. Lam, Y. (2007). The Geometric Process and Its Application. World Scientific Publishing Co. Pvt. Ltd., Singapore.

15. Lone, S. A., Rahman, A. and Islam, A. (2016). A Study of Accelerated Life Testing Design using Geometric Process for Generalized Exponential Distribution using Time Constraint. International Journal of Engineering Science \& Research Technology, 5(2).

16. Pan, Z., Balakrishnan, N. and Sun, Q. (2011.) Bivariate constant-stress accelerated degradation model and inference. Communications in Statistics-Simulation and Computation, 40 (2), 247-257. http://dx.doi.org/10.1080/03610918.2010.534227

17. Rodriguez, R. N. (1977). A guide to Burr Type XII distributions. Biometrika, 64, 129-134.

18. Raqab, M. Z. (1998). Order statistics from the Burr type X model. Computers Mathematics and Applications, $36,111-120$.

19. Rahman, A., Lone, S. A. and Alam, I. (2016). Application of Geometric Process for Generalized Exponential Distribution in Accelerated Life Testing with Complete data. International Journal of Scientific \& Engineering Research, 7 (4).

20. Sartawi, H. A. and Abu-Salih, M. S. (1991). Bayes prediction bounds for the Burr type X model. Communications in Statistics - Theory and Methods, 20, 2307-2330.

21. Sindhu, T. N., Hussain, Z. and Aslam, M. (2016). A simulation study of parameters for the censored shifted Gompertz mixture distribution: A Bayesian approach. Journal of Statistics and Management Systems 19 (3), 423-450.

22. Tabassum, N.S., Riaz, M., Aslam, M. and Ahmed, Z. (2016). A Study of Cumulative Quantity Control Chart for a Mixture of Rayleigh Model under a Bayesian Framework. Revista Colombiana de Estadística 39 (2), 185-204. 
23. Tabassum, N.S. and Hussain, Z. (2018). Mixture of two generalized inverted exponential distributions with censored sample: properties and estimation. Statistica Applicata - Italian Journal of Applied Statistics, 30 (3), 373-391.

24. Sindhu, T. N., Hussain, Z. and Aslam, M. (2019). Parameter and reliability estimation of inverted Maxwell mixture model. Journal of Statistics and Management Systems, 22 (3), 459-493.

25. Surles, J. G. and Padgett, W. J. (2001). Inference for reliability and stress-strength for a scaled Burr Type X distribution. Lifetime Data Analysis, 7, 187-200.

26. Wingo, D. R. (1993). Maximum likelihood methods for fitting the Burr Type XII distribution to multiply (progressively) censored life test data. Metrika, 40, 203-210.

27. Watkins, A. J. and John, A. M. (2008). On constant stress accelerated life tests terminated by Type II censoring at one of the stress levels. Journal of Statistical Planning and Inference, 138 (3), 768-786. http://dx.doi.org/10.1016/j.bbr.2011.03.031

28. Yang, G. B. (1994). Optimum constant-stress accelerated life-test plans. IEEE Transactions on Reliability, $43(4), 575-581$.

29. Zhou, K., Shi, Y. M. and Sun, T. Y. (2012). Reliability Analysis for Accelerated Life-Test with Progressive Hybrid Censored Data Using Geometric Process. Journal of Physical Sciences, 16, 133-143. 